\title{
Influência das variações de carga de treinamento resistido sob o método de circuito, sobre o gasto energético em indivíduos treinados e destreinados
}

\author{
Influence of resistant training charge variations under the circuit method, on energy expenditure in \\ trained and destined individuals \\ Influencia de las variaciones de carga de entrenamiento resistentes bajo el método de circuito, sobre \\ el gasto energético en personas capacitadas y destinadas
}

Recebido: 28/03/2021 | Revisado: 04/04/2021 | Aceito: 13/04/2021 | Publicado: 23/04/2021

\begin{abstract}
Jeferson Américo Ancelmo Teixeira ORCID: https://orcid.org/0000-0002-7922-8811 Universidade Federal de São Carlos, Brasil E-mail: jeferson.aat@gmail.com

Karina Kirschner Lopes Teixeira ORCID: https://orcid.org/0000-0001-9159-8084 Universidade Federal de São Carlos, Brasil E-mail: ninaklopes@gmail.com Jorge Camargo Oishi ORCID: https://orcid.org/0000-0003-2909-2052 Universidade Federal de São Carlos, Brasil E-mail: jorgeoishi@hotmail.com

Valter Mariano dos Santos Júnior ORCID: https://orcid.org/0000-0002-2650-9441 Centro Universitário de Votuporanga, Brasil E-mail: profvaltermariano@gmail.com

Jean Cesar Andrade de Souza

ORCID: https://orcid.org/0000-0002-0313-0279 Universidade Federal de São Carlos, Brasil E-mail: jeancesar.andrade@gmail.com

Cássio Mascarenhas Robert-Pires ORCID: https://orcid.org/0000-0002-0843-3113 Universidade de Araraquara, Brasil E-mail: cassio@cefema.com

Rodrigo Ferro Magosso

ORCID: https://orcid.org/0000-0001-7875-4724 Departamento Científico da Bradhon, Brasil E-mail:rmagosso@hotmail.com
\end{abstract}

\begin{abstract}
Resumo
Objetivo: Avaliar o gasto energético (GE) em um protocolo de treinamento resistido, com diferentes volumes de um peso fixo, pelo método em circuito alternado por segmento em homens treinados (TR) e destreinados (DT). Método: Compuseram a amostra, por voluntariado, 18 homens com idade entre 18 e 35 anos. Após serem informados sobre as etapas do projeto, os voluntários, realizaram uma avaliação da composição corporal, seguido dos testes de uma repetição máxima (1RM) e vinte repetições máximas (20RM), e posterior reteste. O protocolo consistiu em 3 sessões de treinamento em circuito com diferentes volumes (12, 16 e 20 repetições), com um peso fixo de 20RM nos exercícios: leg press, supino reto, mesa flexora e puxador frente; com intervalos de 1 minuto entre os exercícios e 2 minutos entre cada volta, totalizando 3 voltas por sessão. Para análise dos dados foi utilizado o software STATISTIC 7, adotando significância de p<0,05. Resultados: Verificou-se que os voluntários TR apresentaram maior trabalho total em relação aos voluntários DT nas sessões de 12 ( $\mathrm{TR}=15820,80 \pm 2396,50$; DT=10381,60 $\pm 2660,374), 16$ (TR=20919,95 $\pm 3219,82$; $\mathrm{DT}=12727,46 \pm 3143,99)$ e 20 repetições $(\mathrm{TR}=21957,24 \pm 3470,21$; DT $=12959,57 \pm 4084,05)$. O mesmo foi observado no GE dos exercícios nas sessões de 12 ( $\mathrm{TR}=85,01 \pm 14,72$; DT=64,34 $\pm 15,43), 16(\mathrm{TR}=111,10 \pm 25,97 ; \mathrm{DT}=74,24 \pm 20,03)$ e 20 repetições $(\mathrm{TR}=145,33 \pm 58,64 ; \mathrm{DT}=78,69 \pm 24,21)$, e no $\mathrm{GE} /$ repetições nas sessões de $12(\mathrm{TR}=0,59 \pm 0,10$; $\mathrm{DT}=0,44 \pm 0,11), 16(\mathrm{TR}=0,59 \pm 0,13 ; \mathrm{DT}=0,43 \pm 0,12)$ e 20 repetições $(\mathrm{TR}=1,10 \pm 0,34 ; \mathrm{DT}=0,43 \pm 0,13)$. Conclusão: $\mathrm{O}$ GE/repetições não foi afetado nas sessões próximas à falha concêntrica nesse protocolo para indivíduos DT, em contrapartida, para o grupo TR, quanto mais próximo da falha maior foi o GE/repetições.
\end{abstract}

Palavras-chave: Exercício físico; Exercícios em circuito; Força muscular; Gasto energético. 


\begin{abstract}
Objective: To evaluate the energy expenditure (EE) in a resistance training protocol with different volumes of a fixed weight by the alternating circuit method by segment in trained (TR) and untrained (UT) men. Method: The sample comprised, by volunteering, 18 men aged between 18 and 35 years. After being informed about the stages of the project, the volunteers carried out an assessment of their body composition, followed by the tests of a maximum repetition $(1 \mathrm{RM})$ and twenty maximum repetitions $(20 \mathrm{RM})$, and later retest. The protocol consisted of three circuit-training sessions with different training volumes (12, 16 and 20 repetitions) of a fixed weight of 20RM in the leg press, bench press, flexor table and front puller exercises, with 1-minute intervals between the exercises and 2 minutes between each lap, totaling 3 laps per session. For data analysis, the STATISTIC 7 software was used, adopting a significance of $\mathrm{p}<0.05$. Results: It was found that the TR volunteers showed greater total work in relation to the UT volunteers in the sessions of $12 \quad(\mathrm{TR}=15820.80 \pm 2396.50 ; \quad \mathrm{UT}=10381.60 \pm 2660.374), \quad 16 \quad(\mathrm{TR}=20919.95 \pm 3219.82$; $\mathrm{UT}=12727.46 \pm 3143.99)$ and 20 repetitions $(\mathrm{TR}=21957.24 \pm 3470.21 ; \mathrm{UT}=12959.57 \pm 4084.05)$. The same was observed in the $\mathrm{EE}$ of the exercise in the sessions of $12(\mathrm{TR}=85.01 \pm 14.72 ; \mathrm{UT}=64.34 \pm 15.43), 16(\mathrm{TR}=111.10 \pm 25.97$; $\mathrm{UT}=74.24 \pm 20.03)$ and 20 repetitions $(\mathrm{TR}=145.33 \pm 58.64 ; \mathrm{UT}=78.69 \pm 24.21)$, and in the $\mathrm{EE} /$ repetitions in sessions of 12 $(\mathrm{TR}=0.59 \pm 0.10 ; \quad \mathrm{DT}=0.44 \pm 0.11), \quad 16 \quad(\mathrm{TR}=0.59 \pm 0.13 ; \quad \mathrm{UT}=0.43 \pm 0.12) \quad$ and 20 repetitions $\quad(\mathrm{TR}=1.10 \pm 0.34$; $\mathrm{UT}=0.43 \pm 0.13)$. Conclusion: The EE/repetitions were not affected in the sessions close to the concentric failure in this protocol for UT individuals, in contrast, for the TR group, the closer to the failure the greater the EE/repetitions.
\end{abstract}

Keywords: Physical exercise; Circuit exercises; Muscle strength; Energy expenditure.

\begin{abstract}
Resumen
Objetivo: Evaluar el gasto energético (GE) en un protocolo de entrenamiento de resistencia con diferentes volúmenes de un peso fijo por el método de circuito alterno por segmento en hombres entrenados (TR) y desentrenados (DT). Método: La muestra estuvo formada, de forma voluntaria, por 18 hombres con edades comprendidas entre los 18 y los 35 años. Luego de ser informados sobre las etapas del proyecto, los voluntarios realizaron una evaluación de su composición corporal, seguida de las pruebas de una repetición máxima (1RM) y veinte repeticiones máximas (20RM), y posteriormente reteste. El protocolo consistió en 3 sesiones de entrenamiento en circuito con diferentes volúmenes de entrenamiento (12, 16 y 20 repeticiones) de un peso fijo de 20RM en los ejercicios de leg press, press de banca, mesa de flexión y tirador frontal, con intervalos de 1 minuto entre los ejercicios y 2 minutos entre cada vuelta, totalizando 3 vueltas por sesión. Para el análisis de los datos se utilizó el software STATISTIC 7, adoptando una significancia de p <0.05. Resultados: Se encontró que los voluntarios TR mostraron mayor trabajo total en relación a los voluntarios DT en las sesiones de $12 \quad(\mathrm{TR}=15820,80 \pm 2396,50 ; \quad \mathrm{DT}=10381,60 \pm 2660,374), \quad 16 \quad(\mathrm{TR}=20919,95 \pm 3219,82$; $\mathrm{DT}=12727,46 \pm 3143,99)$ y 20 repeticiones $(\mathrm{TR}=21957,24 \pm 3470,21 ; \mathrm{DT}=12959,57 \pm 4084,05)$. Lo mismo se observó en el GE del ejercicio en las sesiones de $12 \quad(\mathrm{TR}=85,01 \pm 14,72 ; \quad \mathrm{DT}=64,34 \pm 15,43), \quad 16 \quad(\mathrm{TR}=111,10 \pm 25,97$; $\mathrm{DT}=74,24 \pm 20,03)$ y 20 repeticiones $(\mathrm{TR}=45,33 \pm 58,64 ; \mathrm{DT}=78,69 \pm 24,21)$, y en el $\mathrm{GE} /$ repeticiones en sesiones de 12 $(\mathrm{TR}=0,59 \pm 0,10 ; \quad \mathrm{DT}=0,44 \pm 0,11), \quad 16 \quad(\mathrm{TR}=0,59 \pm 0,13 ; \quad \mathrm{DT}=0,43 \pm 0,12) \quad$ y 20 repeticiones $\quad(\mathrm{TR}=1,10 \pm 0,34$; $\mathrm{DT}=0,43 \pm 0,13)$. Conclusión: Los $\mathrm{GE} /$ repeticiones no se vieron afectados en las sesiones cercanas al fallo concéntrico en este protocolo para los individuos DT, en contraste, para el grupo TR, cuanto más cerca del fallo mayores GE/repeticiones.
\end{abstract}

Palabras clave: Ejercicio físico; Ejercicios de circuito; Fuerza muscular; Gasto de energía.

\title{
1. Introdução
}

O treinamento resistido é caracterizado pela ação muscular realizada por um determinado segmento corporal, com a finalidade de executar movimentos excêntricos, concêntricos ou isométricos contra uma resistência estabelecida através de pesos livres, peso corporal e/ou resistência elástica (Fleck \& Kraemer, 2017). Este tipo de treinamento, para melhor eficiência e eficácia, manipula diferentes variáveis, como: a ordem e seleção dos exercícios, velocidade de execução, intervalo entre as séries, densidade - variável relacionada ao tempo de trabalho e de recuperação; volume - variável influenciada pela duração da sessão, número de repetições, de séries e quantidade de exercícios; e intensidade - variável relacionada ao peso utilizado para a execução do treinamento; a fim de aperfeiçoar os resultados dos indivíduos, seja de forma aguda ou crônica (Fleck \& Kraemer, 2014).

Dentre todas as variáveis do treinamento mencionadas, a intensidade tem se mostrado uma das mais importantes para a eficiência do treinamento, pois através desta pode ser gerado diferentes tipos de estímulos (Fleck \& Kraemer, 2014), que por sua vez podem ser exemplificados pelos treinos submáximos - determinados pela manutenção do peso e potência em todas as séries (Izquierdo et al., 2006; Neto et al., 2017); e treinos máximos - execução até a incapacidade em realizar a mecânica do exercício (Kraemer et al., 1987; Burd et al., 2012), com efeito negativo na manutenção dentro de uma zona de repetições selecionada para a mesma intensidade (ex.: 8 à 12 repetições), e tendência de redução significativa no número de repetições em séries consecutivas 
(Willardson et al., 2006; Silva-Gricoletto et al., 2013). Logo, à medida que a intensidade aumenta, os músculos fadigam mais rapidamente, resultando em um número menor de repetições concluídas (Baechle, Earle \& Wathen, 2015).

Assim, apesar da importância da manipulação das variáveis do treinamento para obtenção dos benefícios à saúde, pouco se tem estudado sobre o gasto energético (GE) para a prescrição do treinamento resistido, tornando relevante a realização de pesquisas que visem elucidar tal relação em prol do emprego assertivo destas variáveis e associação com outros aspectos relevantes à saúde dos indivíduos (Vezina et al., 2014), como por exemplo: à adequação nutricional.

Adicionalmente, entre os métodos de aplicação do treinamento resistido podemos destacar aqueles realizados em circuito - alternância de exercícios seguidos com o mínimo de intervalos entre eles. Este método, através da manipulação das variáveis do treinamento, como: volume e intensidade, permite um aumento efetivo na duração das sessões, ao passo que aciona a via aeróbia, a qual possui importante participação no GE (Fleck \& Kraemer, 2014). Por conseguinte, o GE é comumente caracterizado pela hidrólise de Adenosina Trifosfato (ATP), onde a ação mecânica muscular, frente ao exercício, promove uma demanda energética proveniente desta molécula, posteriormente, ressintetizada à partir de nutrientes contidos nos alimentos, principalmente a glicose (Binzen, Swan \& Manore, 2001; Scoott, 2006; Pinheiro-Volp et al., 2011; Redondo, 2015).

Em continuação, no treinamento resistido, o GE aeróbio é geralmente avaliado por calorimetria indireta, através da análise do volume de oxigênio $\left(\mathrm{O}_{2}\right)$ nos gases expirados durante o exercício, refletindo a oxidação dos nutrientes - carboidratos, lipídeos e pequenas quantidades de proteínas (Green, 1994; Aniceto et al., 2013); sendo o consumo de oxigênio convertido para 4,69 a 5,05 Kcal (quilocalorias) para cada litro de ar expirado (Wilmore \& Costill, 2019).

Por outro lado, para avaliação do GE anaeróbio, a medida mais referida na literatura é o equivalente energético do pico de lactato sanguíneo após o exercício (Reis, 2011). Neste viés, estudos pioneiros realizados por Margaria et al. (1963), subsequentemente completado por Di Prampero (1981), permitiram o estabelecimento de um equivalente energético quantitativo para o acúmulo de lactato pós-exercício no sangue, que poderia ser usado para quantificar a energia produzida pela via anaeróbia, considerando que para cada mmol (milimol) de lactato por Kg (quilograma) de massa corporal existe o consumo de $3 \mathrm{ml}$ (mililitros) de $\mathrm{O}_{2}$, então, realiza-se o cálculo da razão do volume consumido de $\mathrm{O}_{2}$ por 5,05 Kcal. A partir do GE aeróbio e anaeróbio pode-se obter o GE total de uma sessão de treinamento e assim avaliar a eficiência da mesma (Scoott et al., 2011).

Neste cenário, ao comparar dois estudos, verificou-se que em séries submáximas de 50\% de uma repetição máxima (1RM) - teste validado para avaliação da força muscular dinâmica que permite determinar a carga máxima superada em uma repetição; do exercício supino reto com 7, 14 e 21 repetições, tanto o trabalho, quanto o GE total, aumentaram proporcionalmente ao número de repetições (Scoott, Croteau \& Ravlo, 2009) e progressão do treinamento até a falha muscular (Scoott, Leary \& Tenbraak, 2011). Em outro estudo, Gorostiaga et al. (2010) avaliaram duas sessões de treinamento de 10 e de 5 repetições, ambos com o peso equivalente a dez repetições máximas (10RM), e verificaram a existência do aumento do lactato nas últimas 5 repetições e predominância anaeróbia na sessão até a falha concêntrica (10 repetições), e que quando comparado a GE total, a metade da sessão de 10 repetições foi menor que a de 5 repetições.

No entanto, ao compararmos os estudos de Scoott, Croteau e Ravlo (2009) e Gorostiaga et al. (2010), notamos a existência de um ponto de proximidade da falha concêntrica no qual o GE por repetição se torna mais elevado. Diante disso, houve a necessidade de avaliar se o GE em sessões de treinamento resistido com diferentes volumes seria maior quanto mais próximo a falha, tanto em indivíduos destreinados (DT), como em indivíduos treinados (TR). Assim, o objetivo do presente estudo foi avaliar o GE em um protocolo de treinamento resistido, com diferentes volumes de um peso fixo, pelo método em circuito alternado por segmento em homens TR e DT. 


\section{Metodologia}

\subsection{Abordagem experimental do problema}

O presente estudo se caracterizou como uma pesquisa de abordagem quantitativa, do tipo transversal (Pereira et al., 2018). Para isso foi realizado um teste e um reteste de uma repetição máxima (1RM) e de vinte repetições máximas (20RM), para determinação do peso equivalente a 20RM para os exercícios: leg press (LP), supino reto (SR), mesa flexora (MF) e puxador frente (PF); e a partir deste peso foram determinadas as três sessões como 12, 16 e 20 repetições, que foram realizadas com o mesmo peso do teste de 20RM.

\subsubsection{Caracterização dos voluntários}

Participaram do estudo 18 indivíduos adultos, voluntários, do sexo masculino, com idade entre 18 e 35 anos. Estes foram divididos em grupo TR: constituído por 9 indivíduos com mais de um ano de treinamento, idade média de 27,55 44,37, estatura $(\mathrm{cm})$ igual à $173,88 \pm 5,15$, peso corporal $(\mathrm{Kg})$ de 77,77 $\pm 8,78$, Índice de Massa Corporal (IMC) equivalente à 25,76 $\pm 2,85$, percentual (\%) de Massa Gorda (Kg) de 20,42 $\pm 6,54$, e \% de Massa Magra igual a 60,45 $\pm 2,71$; e um grupo DT: formado por 9 indivíduos que não possuíam experiência em treinamento resistido, idade média de $28 \pm 5,78$, estatura $(\mathrm{cm})$ igual à 173,5 $\pm 9,78$,

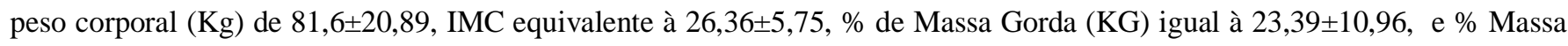

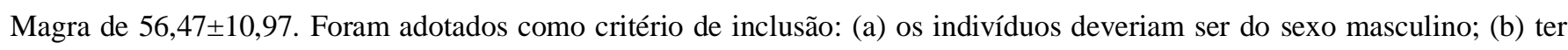
idade entre 18 e 35 anos; (c) praticar de forma regular treinamento resistido por período superior a um ano - grupo TR; e (d) não possuir experiência em treinamento resistido - grupo DT. Por outro lado, os critérios de exclusão adotados foram: (a) uso de esteroides anabólicos androgênicos nos seis meses que precederam o estudo; (b) presença de alguma doença metabólica que pudesse alterar os resultados do estudo; (c) alguma patologia ou lesão que limitasse a execução total ou parcial do protocolo de treinamento; (d) presença de problemas cardíacos, respiratórios, musculares, como alguma lesão ou cirurgia recente; e (e) indivíduos fumantes. Os voluntários foram orientados a manterem o mesmo padrão dietético, evitando alimentos e bebidas estimulantes nas 24 horas que precederam os testes, tão pouco realizar exercícios físicos nos três dias que antecederam cada intervenção, esta que teve os mesmos horários fixados.

\subsection{2 Ética}

Em consonância com as normas que regulamentam a pesquisa com seres humanos (Resolução 466/12 do Conselho Nacional de Saúde), este projeto foi submetido e aprovado pelo Comitê de Ética e Pesquisa em Seres Humanos da Universidade Federal de São Carlos - UFSCar (CAAE: 59303416.1.0000.5504). Todos os procedimentos foram realizados no Laboratório de Fisiologia do Exercício do Departamento de Ciências Fisiológicas da UFSCar, na cidade de São Carlos, São Paulo, Brasil.

\subsection{Procedimentos Experimentais}

\subsubsection{Avaliações antropométricas}

Na primeira etapa desta pesquisa os voluntários foram convidados a comparecerem ao laboratório supramencionado, onde receberam dos pesquisadores informações sobre as fases e procedimentos à serem realizados neste projeto, riscos e benefícios, assim como os objetivos desta pesquisa, devendo assinar o Termo de Consentimento Livre e Esclarecido (TCLE) ao mostrar-se interessado em participar do estudo. Posteriormente, os voluntários foram avaliados fisicamente - peso corporal e estatura - através de uma balança antropométrica mecânica modelo 104 A (Welmy ${ }^{\circledR}$, Santa Barbara D’Oeste, Brasil), com precisão de 100 gramas e capacidade de 300 quilos, e escala de precisão de $0,1 \mathrm{~cm}$. O Índice de Massa Corporal (IMC) foi determinado pela razão do peso pela estatura ao quadrado $\left(\mathrm{IMC}=\right.$ peso/estatura $\left.{ }^{2}\right)$. Também foi realizada a análise da massa corporal por densitometria, utilizando o equipamento DXA (Dual X-ray Absorptiometry). 


\subsubsection{Testes de repetições máximas}

Na segunda etapa da pesquisa os indivíduos realizaram duas sessões de familiarização, afim de refinar o padrão de movimento pré-estabelecido. Concluído este período inicial, os voluntários foram submetidos ao teste de 1RM, segundo Brown e Weir (2001), nos exercícios LP, SR, MF e PF, a partir de um aquecimento de oito repetições com 50\% de 1RM estimada, intervalo de dois minutos de repouso, seguida de uma série com 70\% de 1RM. Após o aquecimento, foram iniciadas as tentativas para determinação de 1RM, estabelecendo o mínimo de três e o máximo de cinco tentativas, com intervalos de cinco minutos entre cada uma delas. Após 48 horas foi realizado o reteste, e se houvesse uma diferença de 5\% entre as tentativas um terceiro teste seria realizado. Para o teste de 20RM nos exercícios LP, SR, MF e PF, foi estimado que o peso de 20RM estaria em torno de 55\% à 60\% de 1RM de acordo com os dados encontrados na literatura (Shimano et al., 2006). Tal teste consistiu em determinar o peso máximo em que o indivíduo conseguiria realizar 20 repetições sem capacidade de realizar a $21^{\mathrm{a}}$ repetição. Caso o voluntário realizasse mais ou menos que 20 repetições, o peso era ajustado, em um total de no máximo duas tentativas com intervalos de cinco minutos. Após 72 horas, realizou-se o reteste, e se houvesse diferença de 5\% entre as tentativas um terceiro teste seria realizado.

\subsubsection{Protocolo de treino em circuito}

Todos os voluntários foram instruídos a manterem o mesmo padrão dietético, evitando alimentos e bebidas estimulantes nas 24 horas que precederam os testes tão pouco realizar exercícios 72 horas antes dos testes. Todas as sessões foram realizadas nos mesmos horários. Após os testes de RM, os voluntários passaram por três sessões de treinamento com diferentes volumes (12, 16 e 20 repetições) de um peso fixo de 20RM. As sessões foram estipuladas por sorteio, contendo um intervalo de 72 horas entre elas. O protocolo utilizado nas sessões de treinamento foi o método em circuito alternado por segmento, sendo os exercícios realizados na seguinte sequência: LP, SR, MF e PF; com intervalos de 1 minuto entre os exercícios e 2 minutos entre cada volta, totalizando 3 voltas por sessão.

\subsubsection{Lactacidemia}

Para a análise da lactacidemia foi coletada uma amostra de $25 \mu \mathrm{L}$ (microlitros) de sangue do lobo da orelha do voluntário e armazenada em um microtubo contendo $50 \mu \mathrm{L}$ (microlitros) de fluoreto de sódio a $1 \%$, e posteriormente foram realizadas as análises por meio de um lactímetro eletroenzimático do Laboratório de Fisiologia do Exercício da Universidade Federal de São Carlos - UFSCar. As coletas foram realizadas pré e 5 minutos após a sessão de cada treino, para que fosse calculada a diferença entre esses dois pontos ( $\Delta \mathrm{lac}$ ) e este foi utilizado para o cálculo do componente anaeróbio do gasto energético.

\subsubsection{Gasto Energético (GE)}

Com relação ao GE, para a quantificação do componente aeróbio, foram realizadas as coletas de gases expirados por um analisador portátil de gases VO2000 Aerosport ${ }^{\circledR}$ (Medgraphic $^{\circledR}$, Saint Paul, Minnesota, United States of America), com pneumotacógrafo de fluxo baixo (até $70 \mathrm{l} / \mathrm{min}$ ) previamente calibrado de acordo com as instruções do fabricante. Os dados foram analisados pelo software Aerograph ${ }^{\circledR}\left(\right.$ Medgraphic $^{\circledR}$, Saint Paul, Minnesota, United States of America), em modo de coleta com média a cada 20 segundos. Antes do início das coletas, os indivíduos permaneceram com a máscara e em repouso, enquanto o software não fazia a gravação dos dados. Após estes 10 minutos iniciais, destinados ao ajuste ventilatório e retorno ao metabolismo de repouso (Aliasgharzadeh et al., 2015), eram gravados 5 minutos para o cálculo do consumo de repouso.

O componente aeróbio foi calculado a partir do GE total obtido pelo software, desconsiderando o GE do repouso, multiplicado pelo tempo total da sessão, visto que este gasto todos os voluntários iriam ter, independentemente de estarem 
executando as sessões, conforme a equação à seguir: [Componente Aeróbio = GE total - (Média do GE repouso × Tempo Total)]. O componente anaeróbio foi calculado pelo produto da variação na lactacidemia $(\Delta \mathrm{lac})$ pelo peso corporal em quilos, em seguida

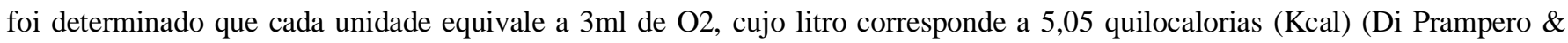
Ferretti, 1999), conforme a equação: [Componente Anaeróbio $=(\Delta$ lac. $\times$ Peso Corporal $\times 0,003 \times 5,05)]$. Já o GE total da sessão foi calculado pela soma dos componentes aeróbio e anaeróbio de cada sessão de treinamento e descrito nas unidades de KCal. A quantidade de energia por repetição (KCal/repetições) foi calculada por meio da seguinte equação: [GE/Repetição $(\mathrm{KCal} /$ repetições $)=$ GE Exercício/Repetições] .

\subsection{Análise estatística}

Os dados foram tratados por procedimentos estatísticos compatíveis com os objetivos propostos, utilizando o software estatístico STATISTIC 7 (Statsoft inc. Tulsa. USA). Para as análises das diferenças das sessões de cada grupo foi realizado primeiramente a verificação da normalidade dos dados pelo teste de Kolmogorov-Smirnov e homogeneidade por Levene. Os testes posteriores foram realizados de acordo com a normalidade dos dados, teste de variância Anova Two-Way, e para identificar onde as diferenças ocorreram, foi utilizado o teste de Post-hoc Tukey para as variáveis paramétricas e Kruskal Wallis Anova para as variáveis não paramétricas. Já para as comparações entre os grupos nos testes de 1RM e 20RM, foi utilizado o teste $t$ de Student. Para as conclusões das análises estatísticas foi utilizado o nível de significância de 5\% ( $<<0,05)$.

\section{Resultados}

De acordo com o cálculo amostral proposto por Cohen (1992): $\left[n=N . Z^{2} \cdot p \cdot(1-p) / Z^{2} \cdot p \cdot(1-p)+e^{2} \cdot N-1\right]$, sendo $n$ a amostra calculada, $N$ a população, $Z$ a variável normal, $p$ a real probabilidade do evento, e $e$ o erro amostral; e em consonância com Santos (2017), para um nível padrão $\alpha$ de 0,05 o poder da amostra recomendado foi de 0,80 , e a partir deste cálculo, o resultado exigido para o presente estudo foi de 8 voluntários por grupo para um tamanho do efeito de 0,60 (poder da amostra 0,9135713).

De modo geral, analisando os dados referentes a tabela 1 na qual estão expressos em média e desvio padrão os resultados obtidos pelos grupos TR e DT nos testes de 1RM e 20RM, assim como os resultados comparativos entre os grupos realizado por meio do teste $t$ de Student, verificou-se uma diferença significativa entre os grupos TR e DT nos exercícios LP, SR e PF tanto no teste de 1RM, quanto no teste de 20RM, sendo o grupo TR aquele com escores médios maiores.

Tabela 1. Comparação das médias \pm desvio padrão nos testes de 1RM e 20RM nos respectivos exercícios físicos entre os grupos DT e TR.

\section{$1 \mathrm{RM}(\mathrm{Kg})$}

\begin{tabular}{lcccc}
\hline Exercícios & Grupo DT $(\mathbf{n = 9})$ & Grupo TR $(\mathbf{n = 9})$ & Grupo DT $(\mathbf{n = 9})$ & Grupo TR $(\mathbf{n = 9})$ \\
\hline Leg Press & $283,89 \pm 69,18^{*}$ & $411,11 \pm 59,47^{*}$ & $196,67 \pm 48,09^{*}$ & $306,67 \pm 50,81^{*}$ \\
Supino Reto & $56,94 \pm 14,84^{*}$ & $97,89 \pm 13,53^{*}$ & $31,29 \pm 8,74^{*}$ & $54,69 \pm 6,99 *$ \\
Mesa Flexora & $51,96 \pm 18,56$ & $66,67 \pm 11,55$ & $25,44 \pm 10,48$ & $33,78 \pm 7,45$ \\
Puxador Frente & $59,89 \pm 12,35^{*}$ & $77,44 \pm 10,55^{*}$ & $32,78 \pm 6,72^{*}$ & $44,22 \pm 6,42^{*}$ \\
\hline
\end{tabular}

Legenda: RM: repetições máximas; Kg: quilogramas; TR: treinados; DT: destreinados; n: número de voluntários. Os dados estão apresentados em média \pm desvio padrão. Para a comparação dos grupos nos diferentes exercícios de acordo com os testes de repetição máxima foi utilizado o teste t de Student. * $p<0,05$, diferença entre DT e TR.

Fonte: Autores.

Mediante a Tabela 2, que apresenta o número de repetições realizadas para cada sessão em ambos os grupos, assim como o trabalho total, observou-se diferenças significativas entre os grupos DT e TR em repetições realizadas na sessão de 16 
repetições e no trabalho total nas sessões de 12, 16 e 20 repetições. Quanto ao número de repetições realizadas, foram encontradas diferenças significativas entre as sessões de 12 e 20 repetições nos grupos DT e TR, e entre as sessões de 12 e 16 repetições no grupo TR. Já em relação ao trabalho total houve diferenças estatisticamente significativas entre as sessões de 12 e 16 repetições, e 12 e 20 repetições apenas no grupo TR.

Tabela 2. Número de repetições realizadas (soma das repetições durante as 3 voltas) e trabalho total em quilos (peso de 20RM $\times \mathrm{n}^{\mathbf{o}}$ de repetições da sessão) dos grupos DT e TR nas respectivas sessões de treinamento resistido, valores expressos em média \pm desvio padrão.

\begin{tabular}{lcccc}
\hline & \multicolumn{2}{c}{ Repetições Realizadas } & \multicolumn{2}{c}{ Trabalho Total (Kg) } \\
\hline $\begin{array}{l}\text { Sessões } \\
\left(\mathbf{n}^{\circ} \text { repetições }\right)\end{array}$ & Grupo DT $(\mathbf{n = 9})$ & Grupo TR $(\mathbf{n = 9})$ & Grupo DT $(\mathbf{n = 9 )}$ & Grupo TR $(\mathbf{n = 9 )}$ \\
\hline $\mathbf{1 2}$ repetições & $144,00 \pm 0,00^{+}$ & $144,00 \pm 0,00^{\#+}$ & $10381,60 \pm 2660,374 *$ & $15820,80 \pm 2396,50 * \#+$ \\
$\mathbf{1 6}$ repetições & $172,00 \pm 18,80^{*}$ & $187,67 \pm 5,50 * *$ & $12727,46 \pm 3143,99 *$ & $20919,95 \pm 3219,82 * \#$ \\
$\mathbf{2 0}$ repetições & $182,33 \pm 23,10^{+}$ & $192,00 \pm 20,18^{+}$ & $12959,57 \pm 4084,05^{*}$ & $21957,24 \pm 3470,21 *+$ \\
\hline
\end{tabular}

Legenda: Kg: quilogramas; DT: destreinados; TR: treinados; n: número. Os dados estão apresentados em média \pm desvio padrão. Para as repetições realizadas foi utilizado o teste não paramétrico Kruskal Wallis. Para o trabalho total foi utilizado o teste paramétrico ANOVA twoway de Post Hoc de Tukey Kramer. * $p<0,05$, diferença entre DT e TR em 12, 16 e 20 repetições. * $p<0,05$, diferença entre DT 12 e DT 16 repetições, e diferença entre TR 12 e TR 16 repetições. ${ }^{+} p<0,05$, diferença entre DT 12 e DT 20 repetições, e diferença entre TR 12 e TR 20 repetições.

Fonte: Autores.

Em continuação, na Tabela 3, onde estão apresentados os resultados do GE repouso, GE total e GE por repetição (KCal/repetições) dos grupos DT e TR nas respectivas sessões de treinamento resistido, observou-se diferenças significativas entre os grupos DT e TR em GE por exercício e GE por repetição (KCal/repetições) na sessão de 20 repetições. O mesmo foi evidenciado entre as sessões de 12 e 20 repetições no grupo TR em relação a variável GE por repetição (KCal/repetições).

Tabela 3. GE repouso, GE total e GE por repetição (KCal/repetições) dos grupos DT e TR nas respectivas sessões de treinamento resistido, valores expressos em média \pm desvio padrão.

\begin{tabular}{lcccccc}
\hline & \multicolumn{2}{c}{ GE Repouso } & \multicolumn{2}{c}{ GE exercício (liquido) } & \multicolumn{2}{c}{ KCal/ Repetições } \\
\hline $\begin{array}{l}\text { Sessões } \\
\text { (n repetições) }\end{array}$ & $\begin{array}{c}\text { Grupo DT } \\
(\mathbf{n = 9})\end{array}$ & $\begin{array}{c}\text { Grupo TR } \\
(\mathbf{n = 9})\end{array}$ & $\begin{array}{c}\text { Grupo DT } \\
(\mathbf{n = 9})\end{array}$ & $\begin{array}{c}\text { Grupo TR } \\
(\mathbf{n = 9})\end{array}$ & $\begin{array}{c}\text { Grupo DT } \\
(\mathbf{n = 9})\end{array}$ & $\begin{array}{c}\text { Grupo TR } \\
(\mathbf{n = 9})\end{array}$ \\
\hline $\mathbf{1 2}$ repetições & $1,33 \pm 0,30$ & $1,63 \pm 0,32$ & $64,34 \pm 15,43$ & $85,01 \pm 14,72^{+}$ & $0,44 \pm 0,11$ & $0,59 \pm 0,10$ \\
$\mathbf{1 6}$ repetições & $1,34 \pm 0,26$ & $1,85 \pm 0,63$ & $74,24 \pm 20,03$ & $111,10 \pm 25,97$ & $0,43 \pm 0,12$ & $0,59 \pm 0,13$ \\
$\mathbf{2 0}$ repetições & $1,43 \pm 0,14$ & $1,95 \pm 1,07$ & $78,69 \pm 24,21^{*}$ & $145,33 \pm 58,64 *+$ & $0,43 \pm 0,13 *$ & $1,10 \pm 0,34 *$ \\
\hline
\end{tabular}

Legenda: Kg: quilogramas; DT: destreinados; TR: treinados; n: número. Os dados estão apresentados em média \pm desvio padrão. Para o GE repouso e GE por repetição (Kcal/rep.) foi utilizado o teste não paramétrico Kruskal Wallis. Para o GE total foi utilizado o teste paramétrico ANOVA two-way de Post Hoc de Tukey Kramer. ${ }^{*} p<0,05$, diferença entre DT e TR em 12,16 e 20 repetições. ${ }^{+} p<0,05$, diferença entre DT 12 e DT 20 repetições, e diferença entre TR 12 e TR 20 repetições.

Fonte: Autores.

\section{Discussão}

Ao analisar os resultados do GE em um protocolo de treinamento resistido, com diferentes volumes de um peso fixo, pelo método em circuito alternado por segmento em homens TR e DT, os principais achados desta pesquisa demonstraram que o trabalho total do grupo TR foi superior em todas as sessões (12, 16 e 20 repetições) quando comparada ao grupo DT. Além 
disso, para o grupo TR a sessão de 20 repetições apresentou maior trabalho total que a sessão de 12 repetições. Esta diferença pode ter ocorrido pelo fato dos indivíduos TR levantarem maior quantidade de peso e realizarem um maior número de repetições, devido sua capacidade de maior recrutamento muscular dos músculos agonistas e sinergistas diante de repetições que se aproximam da falha concêntrica, fato não observado nos voluntários DT, visto que, logo no início da série estes já possuíam um grande recrutamento muscular, fazendo-os chegar a fadiga mais precocemente.

Tal característica, segundo alguns estudos encontrados na literatura, ocorre em virtude dos indivíduos do grupo DT não possuírem as mesmas adaptações fisiológicas que os voluntários do grupo TR, e assim, diante deste protocolo, apresentaram maiores dificuldades na execução das sessões (Akima et al., 1999; Knight \& Kamen, 2001; Aagaard et al., 2002; Carroll, Riek \& Carson, 2002; Del Vecchio et al., 2019; Santos, Freitas-Júnior \& 2020), e consequentemente atingindo resultados inferiores em todas as variáveis analisadas nesta pesquisa, exemplificados pelos resultados dos testes de 1RM e 20RM. Teixeira et al. (2019) justifica este cenário dizendo que tal característica certifica a distinção entre grupos quanto aos níveis de aptidão física, confirmando a ideia inicial do estudo de analisar indivíduos TR e DT. Por conseguinte, este fato também comprova que realizar exercícios físicos, de modo regular, melhora as funções fisiológicas atribuindo vantagens aos sujeitos TR em relação aos DT (Guerra et al., 2021).

Esses resultados corroboram com o estudo de Sundstrup et al. (2012), que demonstrou em um protocolo de 15RM, através da atividade eletromiográfica, que mulheres DT chegaram a fadiga antes mesmo da falha concêntrica, onde a ativação muscular e maior recrutamento de fibras musculares do tipo IIb - fibras de contração rápida; esteve aumentada nas primeiras repetições e se manteve ao longo da série. Esta fadiga precoce, segundo Eches et al. (2013), reflete na diminuição do desempenho motor no decorrer das séries, ainda que o volume total de treinamento seja alcançado. Logo, o resultado encontrado pelo presente estudo, sugere que os protocolos de treinamento resistido para iniciantes busquem evitar a falha concêntrica, pois estes indivíduos atingem a fadiga antes que ela ocorra, o que prejudica algumas funções fisiológicas, comprometendo a condução do estímulo elétrico despolarizante ao longo de uma unidade motora, culminando na diminuição da força muscular e a manutenção das ações musculares (Souza et al., 2019), e assim, inviabilizando o alcance dos objetivos propostos nos programas de treinamento voltados a esta população.

Continuando, em relação ao GE total do exercício (sem o EPOC - excesso de oxigênio consumido pós-exercício), para o grupo DT, a manutenção do volume nas sessões determinadas (12, 16 e 20 repetições) não resultou em diferença significativa. No entanto, os voluntários TR se mostraram superiores aos DT na sessão de 20 repetições, apresentando maior GE total nas sessões até a falha concêntrica. Ou seja, o maior trabalho total no grupo TR apresentou uma relação com o GE, assim como demonstrado por Scott, Leary e Tenbraak (2011), os quais concluíram em estudo realizado com homens TR, que quanto maior o trabalho total maior é o GE total. Jockner et al. (2019) atribui este GE ao maior recrutamento muscular provocado pelo treinamento resistido, já Alves et al. (2018) o relaciona ao volume total realizado nas sessões de treinamento.

Outrossim, devido a diferença do volume entre as sessões, determinamos nesta pesquisa o GE/repetições, onde os resultados foram similares aos apresentados anteriormente no GE total (TR se mostraram superiores aos voluntários DT na sessão de 20 repetições). Logo, a ativação muscular resultante do padrão de movimento desordenado ou falta de coordenação intramuscular e intermuscular ao longo da sessão para o grupo DT, refletiu na fadiga precoce (Maior \& Alves, 2003; Eches et al., 2013). Adicionalmente, o grupo TR demonstrou maior capacidade de tolerar a acidose metabólica, a qual permitiu que estes indivíduos realizassem mais repetições e consequentemente apresentassem maior GE/repetições (Tiggemann, 2007).

Por fim, indagamos que pesquisas futuras sejam realizadas com intuito de melhor esclarecer o GE em diferentes tipos, métodos, intensidades e volumes de treinamento, uma vez que este estudo apresentou algumas limitações, como: diferentes volumes (GE/repetições) e densidades (esforço e recuperação durante o treino). No entanto, Mazzetti et al. (2007) verificaram em três protocolos, com diferentes tempos de contração e intervalo, e volume similar ao presente estudo, diferenças no 
GE/minuto, mas não no GE total. Assim, ressaltamos que esta pesquisa seguiu rigorosamente seu objetivo, de modo a ofertar resultados relevantes a temática abordada, contribuindo para a realização de futuros estudos e intervenções.

\section{Conclusão}

Com base nos resultados encontrados, concluímos que o GE/repetições não foi afetado nas sessões próximos da falha concêntrica nesse protocolo para indivíduos DT, ao passo que para o grupo TR, quanto mais próximo da falha concêntrica maior foi o GE/repetições. Finalmente, para que possamos compreender melhor os efeitos dos protocolos de exercício resistido no GE, produzindo informações relevantes à temática abordada e à prescrição do treinamento, sugerimos aos futuros estudos a ampliação da amostra, incluindo voluntários do sexo masculino e feminino, e um maior controle do volume e da densidade de treinamento.

\section{Aplicações Práticas}

Para indivíduos DT que objetivam maior GE uma estratégia eficiente pode ser o aumento do número de séries ou mesmo de exercícios, pois os resultados apresentados neste estudo demonstraram que o aumento do número de repetições por séries não foi efetivo. Por outro lado, para indivíduos TR o aumento do número de repetições por séries pode ser uma estratégia eficiente para o aumento do GE.

\section{Agradecimentos}

A todos aqueles que colaboraram com a realização deste estudo, em especial ao Prof. Dr. Sérgio Eduardo de Andrade Perez, pelo auxílio na elaboração deste artigo, disponibilização de seu laboratório de pesquisa e alguns recursos materiais importantes à execução deste trabalho.

\section{Referências}

Aagaard, P., Simonsen, E. B., Andersen, J. L., Magnusson, P., \& Dyhre-Poulsen, P. (2002). Increased rate of force development and neural drive of human skeletal muscle following resistance training. Journal of applied physiology, 93(4), 1318-1326. https://doi.org/10.1152/japplphysiol.00283.2002

Akima, H., Takahashi, H., Kuno, S. Y., Masuda, K., Masuda, T., Shimojo, H., Anno, I., Itai, Y., \& Fiatsuta, S. (1999). Early phase adaptations of muscle use and strength to isokinetic training. Medicine and science in sports and exercise, 31(4), 588-594. https://doi.org/10.1097/00005768-199904000-00016

Aliasgharzadeh, S., Mahdavi, R., Jafarabadi, M. A., \& Namazi, N. (2015). Comparison of indirect calorimetry and predictive equations in estimating resting metabolic rate in underweight females. Iranian journal of public health, 44(6), 822-829. Retirado em https://www.ncbi.nlm.nih.gov/pmc/articles/PMC4524307/

Alves, R. C., Prestes, J., Bueno, J. C. A., Del Vecchio, F. B., \& Junior, T. P. S. (2018). Comparação do gasto energético em diferentes métodos do treinamento de força. ConScientiae Saúde, 17(3), 293-301. https://doi.org/10.5585/conssaude.v17n3.8288

Aniceto, R. R., Ritti-Dias, R. M., Scott, C. B., Lima, F. F. M. D., Prazeres, T. M. P. D., \& Prado, W. L. D. (2013). Efeitos agudos de diferentes métodos de treinamento com pesos sobre o gasto energético em homens treinados. Revista Brasileira de Medicina do Esporte, 19(3), 181-185. Retirado em https://www.scielo.br/pdf/rbme/v19n3/07.pdf

Baechle, T. R., Earle, R. W., \& Wathen, D. (2015). Resistance training. In: Haff, G. G., \& Triplett, N. T. Essentials of Strength Training and Conditioning. (4a ed). Edition Champaign, Illinois: Human Kinetics Publishers. p 395- 425.

Binzen, C. A., Swan, P. D., \& Manore, M. M. (2001). Postexercise oxygen consumption and substrate use after resistance exercise in women. Medicine and science in sports and exercise, 33(6), 932-938. https://doi.org/10.1097/00005768-200106000-00012

Brown, L. E., \& Weir, J. P. (2001). Asep Procedures Recommendation I - Accurate Assessment of Muscular Strength and Power. Journal of Exercise Physiology Online. 4(3), 1-21.

Burd, N. A., Andrews, R. J., West, D. W., Little, J. P., Cochran, A. J., Hector, A. J., ... \& Phillips, S. M. (2012). O tempo do músculo sob tensão durante o exercício de resistência estimula as respostas sintéticas subfacionárias diferenciais da proteína muscular nos homens. The Journal of physiology, 590(2), 351362. https://doi.org/10.1113/jphysiol.2011.221200

Carroll, T. J., Riek, S., \& Carson, R. G. (2002). The sites of neural adaptation induced by resistance training in humans. The Journal of physiology, 544(2), 641652. https://doi.org/10.1113/jphysiol.2002.024463

Cohen, J. (1992). A power primer. Psychological Bulletin, 112(1), 155-159. https://doi.org/10.1037/0033-2909.112.1.155 
Del Vecchio, A., Casolo, A., Negro, F., Scorcelletti, M., Bazzucchi, I., Enoka, R., ... \& Farina, D. (2019). The increase in muscle force after 4 weeks of strength training is mediated by adaptations in motor unit recruitment and rate coding. The Journal of physiology, 597(7), 1873-1887. https://doi.org/ 10.1113/JP277250

Di Prampero, P. E. (1981). Energética do exercício muscular. Reviews of Physiology, Biochemistry and Pharmacology, 89, 143-222. https://doi.org/10.1007/BFb0035266

Di Prampero, P. E., \& Ferretti, G. (1999). The energetics of anaerobic muscle metabolism: a reappraisal of older and recent concepts. Respiration physiology, 118(3), 103-115. https://doi.org/10.1016/S0034-5687(99)00083-3

Eches, E. H. P., Ribeiro, A. S., Nascimento, M. A., \& Cyrino, E. S. (2013). Desempenho motor em séries múltiplas até a falha concêntrica. Motriz: Revista de Educação Física, 19(3), 43-48. https://doi.org/10.1590/S1980-65742013000700007

Fleck, S. J., \& Kraemer, W. J. (2014). Designing Resistance Training Programs. (4a ed). Editon Champaign, Illinois: Human Kinetics Publishers.

Fleck, S. J., \& Kraemer, W. J. (2017). Fundamentos do treinamento de força muscular. (4a ed). Artmed.

Gorostiaga, E. M., Navarro-Amezqueta, I., Cusso, R., Hellsten, Y., Calbet, J. A., Guerrero, M., \& Izquierdo, M. (2010). Anaerobic energy expenditure and mechanical efficiency during exhaustive leg press exercise. PloS one, 5(10), e13486. https://doi.org/10.1371/journal.pone.0013486

Green, J. H. (1994). Assessment of energy requirements. In: Heatley, R. V., Green, J. H., \& Losowsky, M. S. Consensus in clinical nutrition. (1a ed). Cambridge: Cambridge University Press. p. 22- 37.

Guerra, M. F. S. S., Souza, J. P., Porto, M. J., Araujo, A. M. B., Nascimento, M. B., Andrade, W. B., Santana, W. N. B., Santos, G. P., Santana, A. F., ... \& Silva, S. R. S. (2021). Contribuições da atividade física no envelhecimento do idoso. Research, Society and Development, [S. 1.],10(1), e11310111537. https://doi.org/10.33448/rsd-v10i1.11537

Izquierdo, M., Ibanez, J., González-Badillo, J. J., Häkkinen, K., Ratamess, N. A., Kraemer, W. J., \& Gorostiaga, E. M. (2006). Differential effects of strength training leading to failure versus not to failure on hormonal responses, strength, and muscle power gains. Journal of applied physiology, $100(1)$, $1647-1656$. https://doi.org/10.1152/japplphysiol.01400.2005

Jockner, M. R., Souza Cavina, A. P., Junior, A. C., Micheletti, J. K., Takahama, B. R., Junior, J. N., \& Vanderlei, F. M. (2019). Correlação entre taxa metabólica basal e variabilidade da frequência cardíaca no treinamento resistido funcional em indivíduos com síndrome metabólica. Colloquium Vitae, 11(1), 45-50. https://doi.org/10.5747/cv.2019.v11.n1.v252

Knight, C. A., \& Kamen, G. (2001). Adaptations in muscular activation of the knee extensor muscles with strength training in young and older adults. Journal of Electromyography and Kinesiology, 11(6), 405-412. https://doi.org/10.1016/S1050-6411(01)00023-2

Kraemer, W. J., Noble, B. J., Clark, M. J., \& Culver, B. W. (1987). Physiologic responses to heavy-resistance exercise with very short rest periods. International 10ornal of sports medicine, 8(04), 247-252. https://doi.org/10.1055/s-2008-1025663

Maior, A. S., \& Alves, A. (2003). A contribuição dos fatores neurais em fases iniciais do treinamento de força muscular: uma revisão bibliográfica. Motriz, 9(3), 161-8. http://files.agenorjunqueira.webnode.com.br/200000083-9dd779e557/Contribui\%C3\%A7\%C3\%A3o\%20dos\%20fatores\%20neurais\%20no\%2 0treino\%20de\%20for\%C3\%A7a.pdf

Margaria, R., Cerretelli, P., Aghemo, P., \& Sassi, G. (1963). Custo de energia para funcionar. Jornal de fisiologia aplicada, 18(2), 367-370. https://doi.org/10.1152/jappl.1963.18.2.367

Mazzetti, S., Douglass, M., Yocum, A., \& Harber, M. (2007). Effect of explosive versus slow contractions and exercise intensity on energy expenditure. Medicine and science in sports and exercise, 39(8), 1291-1301. https://doi.org/ IO.1249/mss.ObOI3e318058a603

Neto, V. G. C., Benteso, C. M., Neto, G. D. A. M., \& Miranda, H. (2017). Hipotensão e variabilidade da frequência cardíaca pó s-exercício de força executado de forma máxima e submáxima. Motricidade, 13(1), 19-29. https://doi.org/10.6063/motricidade.6476

Pereira, A. S., Shitsuka, D. M., Parreira, F. J., \& Shitsuka, R. (2018). Metodologia da pesquisa científica. UFSM. https://repositorio.ufsm.br/bitstream/handle/1/15824/Lic_Computacao_Metodologia-Pesquisa-Cientifica.pdf?sequence=1.

Pinheiro-Volp, A. C., Oliveira, F. C., Duarte Moreira Alves, R., Esteves, E. A., \& Bressan, J. (2011). Energy expenditure: components and evaluation methods. Nutricion hospitalaria, 26(3), 430-440. https://doi.org/10.1590/S0212-16112011000300002.

Redondo, R. B. (2015). Resting energy expenditure; assessment methods and applications. Nutricion hospitalaria,31(3), 245-253. https://doi.org/10.3305/nh.2015.31.sup3.8772.

Reis, V. M. (2011). Gasto energético, custo energético aeróbio e custo energético anaeróbio. Revista Brasileira de Cineantropometria \& Desempenho Humano, 13(6), 484-487. https://doi.org/10.5007/1980-0037.2011v13n6p484

Santos, G. E. O. (2017). Cálculo amostral: calculadora online. http://www.calculoamostral.vai.la

Santos, R. F. D., Freitas-Júnior, W. M. D., \& Araújo, R. O. D. (2020). Avaliação do índice de fadiga muscular de flexores e extensores de joelho em indivíduos ativos e sedentários. Revista Brasileira de Ciências do Esporte, 42:e2008. https://doi.org/10.1016/j.rbce.2018.10.002

Scott, C. B. (2006). Contribution of blood lactate to the energy expenditure of weight training. The Journal of Strength and Conditioning Research, 20(2), 404411. https://journals.lww.com/nsca-jscr/Abstract/2006/05000/CONTRIBUTION_OF_BLOOD_LACTATE_TO_THE_ENERGY.29.aspx

Scott, C. B., Croteau, A., \& Ravlo, T. (2009). Energy expenditure before, during, and after the bench press. The Journal of Strength \& Conditioning Research, 23(2), 611-618. https://doi.org/10.1519/JSC.0b013e31818c2845 
Scott, C. B., Leary, M. P., \& TenBraak, A. J. (2011). Energy expenditure characteristics of weight lifting: 2 sets to fatigue. Applied Physiology, Nutrition, and Metabolism, 36(1), 115-120. https://doi.org/10.1139/H10-093

Scott, C. B., Leighton, B. H., Ahearn, K. J., \& McManus, J. J. (2011). Aerobic, anaerobic, and excess postexercise oxygen consumption energy expenditure of muscular endurance and strength: 1-set of bench press to muscular fatigue. The Journal of Strength \& Conditioning Research,25(4), 903-908. https://doi.org/10.1519/JSC.0b013e3181c6a128

Shimano, T., Kraemer, W. J., Spiering, B. A., Volek, J. S., Hatfield, D. L., Silvestre, R., \& Häkkinen, K. (2006). Relationship between the number of repetitions and selected percentages of one repetition maximum in free weight exercises in trained and untrained men. The Journal of Strength \& Conditioning Research, 20(4), 819-823. Retirado em https://highfit.com.br/wp-content/uploads/2016/12/O-maior-erro-do-personal-I.pdf

Silva-Grigoletto, M. E. D., Valverde-Esteve, T., Brito, C. J., \& García-Manso, J. M. (2013). Capacidade de repetição da força: efeito das recuperações interséries. Revista Brasileira de Educação Física e Esporte, 27(4), 689-705. https://www.scielo.br/pdf/rbefe/2013nahead/aop_2013.pdf

Souza, R. M. P., Lopes, J. E. G., Bernardo, L. D. M., Gouveia, R. G., Leite Filho, M. A., Montenegro, R. C., \& Borges, L. P. N. C. (2019). Análise dos estimadores de fadiga do eletromiograma durante o treinamento resistido de elevada intensidade executado sob diferentes intervalos de séries. RBPFEX-Revista Brasileira de Prescrição e Fisiologia do Exercício, 13(82), 343-350. http://www.rbpfex.com.br/index.php/rbpfex/article/view/1718/1206

Sundstrup, E., Jakobsen, M. D., Andersen, C. H., Zebis, M. K., Mortensen, O. S., \& Andersen, L. L. (2012). Muscle activation strategie s during strength training with heavy loading vs. repetitions to failure. The Journal of Strength \& Conditioning Research,26(7), 1897-1903. https://doi.org/10.1519/JSC.0b013e318239c38e

Teixeira, J. A. A., Magosso, R. F., Pires, C. M. R., Teixeira, K. K. L., Baldissera, V., \& de Andrade Perez, S. E. (2019). Determinação dos níveis de aptidão física para indivíduos treinados e destreinados a partir do Indice de Força Máxima Relativa (IFMR). RBPFEX-Revista Brasileira de Prescrição e Fisiologia do Exercício, 13(82), 182-188. http://www.rbpfex.com.br/index.php/rbpfex/article/view/1585

Tiggemann, C. L. (2007). Comportamento da percepção de esforço em diferentes cargas de exercícios de força em adultos sedentários, ativos e treinados. Dissertação de Mestrado, Programa de Pós-Graduação em Ciências do Movimento Humano. Universidade Federal do Rio Grande do Sul, Porto Alegre, Brasil. https://www.lume.ufrgs.br/bitstream/handle/10183/12901/000634742.pdf?sequence=1\&isAllowed=y

Vezina, J. W., Der Ananian, C. A., Campbell, K. D., Meckes, N., \& Ainsworth, B. E. (2014). An examination of the differences between two methods of estimating energy expenditure in resistance training activities. The Journal of Strength \& Conditioning Research,28(4), 1026-1031. https://doi.org/10.1519/JSC.0000000000000375

Willardson, J. M., Burkett, L. N. (2006). The effect of rest interval length on bench press performance with heavy vs. light loads. The Journal of Strength \& Conditioning Research, 20: 396-9, 2006. 20(2), 396-399. https://doi.org/10.1519/R-17735.1.

Wilmore, J., \& Costill, D. (2019). Physiology of Sports and Exercise. (7a ed). Human Kinetics Publishers. 